\title{
Crystal structure of $N, N^{\prime}$-ethylene-bis(1,3-dimethyl-5-pyrazolethiolato- iminomethyl)copper(II), $\mathrm{C}_{14} \mathrm{H}_{18} \mathrm{CuN}_{6} \mathrm{~S}_{2}$
}

\author{
A. la Cour ${ }^{1}$, H. Toftlund ${ }^{1}$, K. S. Murray ${ }^{\mathrm{I}}$ and E. R. T. Tiekink*,Ill,1 \\ 1 University of Southern Denmark, Main Campus: Odense University, Department of Chemistry, DK-5230 Odense M, Denmark \\ II Monash University. School of Chemistry. PO Box 23. Clayton. Victoria 3800. Australia \\ 111 The University of Adelaide, Department of Chemistry, Australia 5005
}

Received March 22, 2002, accepted and available on-line May 10, 2002; CCDC-No. 1267/828

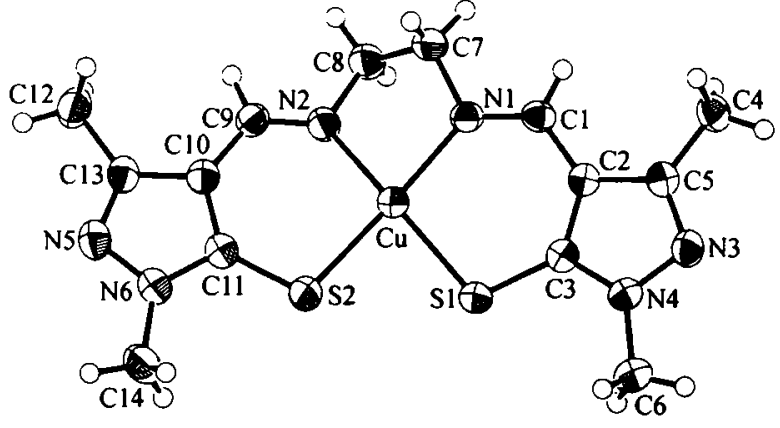

Abstract

$\mathrm{C}_{14} \mathrm{H}_{18} \mathrm{CuN}_{6} \mathrm{~S}_{2}$, triclinic, $P \overline{1}$ (No. 2), $a=8.474(1) \AA, b=12.417(1) \AA$, $c=8.2077(9) \AA, \alpha=99.115(7)^{\circ}, \beta=102.094(9)^{\circ}, \gamma=91.875(9)^{\circ}$, $V=831.9 \AA^{3}, Z=2, R_{\mathrm{gt}}(F)=0.038, w R_{\mathrm{ref}}\left(F^{2}\right)=0.103, T=293 \mathrm{~K}$.

\section{Source of material}

The title compound was crystallised and characterised as described in $[1,2]$.

\section{Discussion}

The $\mathrm{Cu}$ (II) atom exists in a distorted square planar environment defined by a $\mathrm{N}_{2} \mathrm{~S}_{2}$ donor set. The ethylenediamine portion of the ligand chelates the $\mathrm{Cu}$ atom and the thiolate $\mathrm{S}$ atoms derived from the side arms complete the coordination geometry. There are distortions from the ideal square planar geometry with the S1, S2, N1 and $\mathrm{N} 2$ donor atoms lying $-0.035(1) \AA, 0.041(1) \AA, 0.322(3) \AA$, and $-0.367(4) \AA$ out of their least-squares plane, respectively. The $\mathrm{Cu}$ atom lies $0.0223(5) \AA$ out of this plane. The $\mathrm{Cu}-\mathrm{S}$ (2.2696(9) $\AA \& 2.2781$ (9) $\AA$ ) bond distances are equal within experimental error as are the $\mathrm{Cu}-\mathrm{N}$ bonds $(1.998(2) \AA \&$ $1.984(3) \AA$ ). In terms of angles subtended at $\mathrm{Cu}$, the greatest deviation from the ideal geometry is manifested in the $\mathrm{S} 1-\mathrm{Cu}-\mathrm{N} 2$ angle of $167.37(8)^{\circ}$. Whereas the five-membered chelate ring is puckered as expected, the two six-membered rings are planar to $0.037 \AA$ and $0.059 \AA$, respectively and form a dihedral angle of $15.16(9)^{\circ}$. The near planar $\mathrm{CuN}_{2} \mathrm{~S}_{2}$ chromophore is similar to the related $t$-salen $\mathrm{Cu}$ (II) complex [3]. These complexes were used to develop models for type 1 ("blue") copper sites in copper proteins and type A copper sites in cytochrome $C$ oxidase enzyme, which have a more tetrahedral enviroment [4].
Table 1. Data collection and handling.

\begin{tabular}{ll}
\hline Crystal: & $\begin{array}{l}\text { red-orange block, } \\
\text { size } 0.19 \times 0.19 \times 0.32 \mathrm{~mm}\end{array}$ \\
Wavelength: & Mo $K_{\alpha}$ radiation $(0.71073 \AA)$ \\
$\mu:$ & $15.71 \mathrm{~cm}^{-1}$ \\
Diffractometer, scan mode: & Rigaku AFC6R, $\omega / 2 \theta$ \\
$2 \theta_{\text {max }}$ & $55.2^{\circ}$ \\
$N(h k l)_{\text {measured, }} N(h k l)_{\text {unique: }}:$ & 4089,3829 \\
Criterion for $I_{\text {obs, }} N(h k l)_{\text {gt: }}:$ & $I_{\text {obs }}>2 \sigma\left(I_{\text {obs }}\right), 2987$ \\
$N(\text { param })_{\text {refined: }}$ & 209 \\
Programs: & SHELXS-86 [5], teXsan [6], \\
& PLATON [7], ORTEPII [8] \\
\hline
\end{tabular}

Table 2. Atomic coordinates and displacement parameters (in $\AA^{2}$ ).

\begin{tabular}{llrrrl}
\hline Atom & Site & \multicolumn{1}{l}{$x$} & \multicolumn{1}{l}{$y$} & \multicolumn{1}{l}{$z$} & \multicolumn{1}{l}{$U_{\text {iso }}$} \\
\hline H(1) & $2 i$ & -0.0713 & 0.1376 & 0.6704 & $0.064(3)$ \\
H(4a) & $2 i$ & -0.3741 & -0.1076 & 0.4678 & $0.096(4)$ \\
H(4b) & $2 i$ & -0.3546 & 0.0062 & 0.5867 & 0.096 \\
H(4c) & $2 i$ & -0.2093 & -0.0692 & 0.5953 & 0.096 \\
H(6a) & $2 i$ & -0.3300 & -0.0464 & -0.0793 & 0.096 \\
H(6b) & $2 i$ & -0.1586 & 0.0067 & -0.0753 & 0.096 \\
H(6c) & $2 i$ & -0.3062 & 0.0797 & -0.0760 & 0.096 \\
H(7a) & $2 i$ & 0.0437 & 0.3593 & 0.8063 & $0.080(3)$ \\
H(7b) & $2 i$ & 0.1177 & 0.2489 & 0.8445 & 0.080 \\
H(8a) & $2 i$ & 0.3192 & 0.3965 & 0.8772 & 0.080 \\
H(8b) & $2 i$ & 0.3467 & 0.2919 & 0.7530 & 0.080 \\
H(9) & $2 i$ & 0.3848 & 0.5357 & 0.7695 & 0.064 \\
H(12a) & $2 i$ & 0.5341 & 0.8205 & 0.7089 & 0.096 \\
H(12b) & $2 i$ & 0.5789 & 0.7142 & 0.7837 & 0.096 \\
H(12c) & $2 i$ & 0.4159 & 0.7663 & 0.8007 & 0.096 \\
H(14a) & $2 i$ & 0.3262 & 0.7246 & 0.1160 & 0.096 \\
H(14b) & $2 i$ & 0.1656 & 0.6501 & 0.0796 & 0.096 \\
H(14c) & $2 i$ & 0.3279 & 0.5981 & 0.0595 & 0.096 \\
\hline
\end{tabular}

\footnotetext{
* Correspondence author (e-mail: chmtert@nus.edu.sg)

1 Current address: National University of Singapore, Department of Chemistry, Singapore 117543
} 
Table 3. Atomic coordinates and displacement parameters (in $\AA^{2}$ ).

\begin{tabular}{|c|c|c|c|c|c|c|c|c|c|c|}
\hline Atom & Site & $x$ & $y$ & $z$ & $U_{11}$ & $U_{22}$ & $U_{33}$ & $U_{12}$ & $U_{13}$ & $U_{23}$ \\
\hline $\mathrm{Cu}$ & $2 i$ & $0.10687(4)$ & $0.33067(3)$ & $0.42713(4)$ & $0.0299(2)$ & $0.0315(2)$ & $0.0310(2)$ & $0.0046(1)$ & $0.0061(1)$ & $0.0062(1)$ \\
\hline$S(1)$ & $2 i$ & $-0.0162(1)$ & $0.21405(7)$ & $0.18916(9)$ & $0.0556(5)$ & $0.0438(4)$ & $0.0311(4)$ & $-0.0090(4)$ & $0.0073(3)$ & $0.0068(3)$ \\
\hline$S(2)$ & $2 i$ & $0.1535(1)$ & $0.44043(7)$ & $0.2422(1)$ & $0.0633(6)$ & $0.0393(4)$ & $0.0361(4)$ & $-0.0064(4)$ & $0.0041(4)$ & $0.0095(3)$ \\
\hline $\mathbf{N}(1)$ & $2 i$ & $0.0374(3)$ & $0.2494(2)$ & $0.5945(3)$ & $0.036(1)$ & $0.039(1)$ & $0.031(1)$ & $0.002(1)$ & $0.009(1)$ & $0.004(1)$ \\
\hline $\mathbf{N}(2)$ & $2 i$ & $0.2558(3)$ & $0.4131(2)$ & $0.6314(3)$ & $0.039(2)$ & $0.041(1)$ & $0.037(1)$ & $-0.003(1)$ & $0.006(1)$ & $0.009(1)$ \\
\hline $\mathbf{N}(3)$ & $2 i$ & $-0.2972(3)$ & $-0.0327(2)$ & $0.2287(3)$ & $0.038(2)$ & $0.040(1)$ & $0.044(1)$ & $-0.005(1)$ & $0.006(1)$ & $0.006(1)$ \\
\hline$N(5)$ & $2 i$ & $0.3981(3)$ & $0.7216(2)$ & $0.4213(4)$ & $0.039(2)$ & $0.036(1)$ & $0.054(2)$ & $0.001(1)$ & $0.016(1)$ & $0.007(1)$ \\
\hline $\mathbf{N}(6)$ & $2 i$ & $0.3121(3)$ & $0.6398(2)$ & $0.2995(3)$ & $0.038(2)$ & $0.038(1)$ & $0.044(1)$ & $0.005(1)$ & $0.013(1)$ & $0.012(1)$ \\
\hline$C(1)$ & $2 i$ & $-0.0553(4)$ & $0.1621(2)$ & $0.5734(4)$ & $0.031(2)$ & $0.041(2)$ & $0.033(1)$ & $0.003(1)$ & $0.010(1)$ & $0.006(1)$ \\
\hline$C(2)$ & $2 i$ & $-0.1353(3)$ & $0.0997(2)$ & $0.4167(3)$ & $0.026(1)$ & $0.032(1)$ & $0.035(1)$ & $0.005(1)$ & $0.007(1)$ & $0.006(1)$ \\
\hline$C(3)$ & $2 i$ & $-0.1246(3)$ & $0.1156(2)$ & $0.2544(4)$ & $0.029(2)$ & $0.033(1)$ & $0.036(1)$ & $0.006(1)$ & $0.006(1)$ & $0.006(1)$ \\
\hline$C(4)$ & $2 i$ & $-0.3005(4)$ & $-0.0457(3)$ & $0.5218(4)$ & $0.036(2)$ & $0.045(2)$ & $0.048(2)$ & $-0.003(1)$ & $0.011(1)$ & $0.011(1)$ \\
\hline$C(5)$ & $2 i$ & $-0.2442(3)$ & $0.0061(2)$ & $0.3918(4)$ & $0.025(1)$ & $0.036(1)$ & $0.040(2)$ & $0.005(1)$ & $0.007(1)$ & $0.008(1)$ \\
\hline$c(6)$ & $2 i$ & $-0.2571(5)$ & $0.0174(3)$ & $-0.0364(4)$ & $0.069(3)$ & $0.055(2)$ & $0.035(2)$ & $-0.009(2)$ & $0.005(2)$ & $0.002(2)$ \\
\hline $\mathrm{C}(8)$ & $2 i$ & $0.2745(4)$ & $0.3496(3)$ & $0.7699(4)$ & $0.047(2)$ & $0.050(2)$ & $0.038(2)$ & $-0.001(2)$ & $0.003(1)$ & $0.012(1)$ \\
\hline$C(9)$ & $2 i$ & $0.3286(4)$ & $0.5094(2)$ & $0.6595(4)$ & $0.039(2)$ & $0.041(2)$ & $0.036(2)$ & $-0.001(1)$ & $0.008(1)$ & $0.002(1)$ \\
\hline$C(10)$ & $2 i$ & $0.3306(4)$ & $0.5781(2)$ & $0.5390(4)$ & $0.033(2)$ & $0.034(1)$ & $0.040(2)$ & $0.004(1)$ & $0.013(1)$ & $0.004(1)$ \\
\hline$C(11)$ & $2 i$ & $0.2673(4)$ & $0.5526(2)$ & $0.3630(4)$ & $0.031(2)$ & $0.033(1)$ & $0.041(2)$ & $0.009(1)$ & $0.011(1)$ & $0.009(1)$ \\
\hline$C(12)$ & $2 i$ & $0.4919(5)$ & $0.7524(3)$ & $0.7292(5)$ & $0.050(2)$ & $0.044(2)$ & $0.055(2)$ & $-0.008(2)$ & $0.013(2)$ & $-0.002(2)$ \\
\hline$C(13)$ & $2 i$ & $0.4091(4)$ & $0.6844(2)$ & $0.5652(4)$ & $0.030(2)$ & $0.036(2)$ & $0.047(2)$ & $0.002(1)$ & $0.015(1)$ & $0.003(1)$ \\
\hline$C(14)$ & $2 i$ & $0.2802(5)$ & $0.6544(3)$ & $0.1237(4)$ & $0.057(2)$ & $0.059(2)$ & $0.051(2)$ & $-0.003(2)$ & $0.012(2)$ & $0.022(2)$ \\
\hline
\end{tabular}

Acknowledgment. The Australian Research Council is thanked for support.

\section{References}

1. Becher, J.; Toftlund, H.; Olesen, P. H.; Nissen, H.: Preparation of heterocyclic thiolato ligands and their copper(II) complexes. Inorg. Chim. Acta 103 (1985) 167-171.

2. Becher, J.; Toftlund, H.; Olesen, P. H.: A general route to thiolato ligands via tert-butyl sulfides. Chem. Commun. (1983) 740-742.

3. Corrigan, M. F.; Murray, K. S.; West, B. O.; Pilbrow, J. R.: Synthesis and electron spin resonance study of a tetradentate copper(II) mercaptobenzaldimine complex. Aust. J. Chem. 30 (1977) 2455-2463.

4. Anderson, O. P.; Becher, J.; Frydendahl, H.; Taylor, L. F.; Toftlund, H.: Characterization of a pseudotetrahedral copper(II) complex with two thiolato and two imino donor atoms. Chem. Commun. (1986) 699-701.
5. Sheldrick, G. M.: SHELX-86. Program for the automatic solution of crystal structure. University of Göttingen, Germany 1986.

6. teXsan: Single Crystal Structure Analysis Software. Version 1.04. Molecular Structure Corporation. The Woodlands, TX, USA 1997.

7. Spek, T.: PLATON, A Multipurpose Crystallographic Tool, Utrecht University, Utrecht, The Netherlands 2000.

8. Johnson, C. K.: ORTEPII. Report ORNL-5138, Oak Ridge National Laboratory, Tennessee, USA 1976. 